DOI: $10.17234 / S R A Z .65 .12$

\title{
Analyse morphosémantique et comparaison des adjectifs formés avec sub-en français et en croate
}

Marta Petrak

Faculté de philosophie et lettres, Université de Zagreb mpetrak@ffzg.hr

Cette contribution se propose d'explorer et de comparer les adjectifs dérivés à l'aide du préfixe $s u b$ - en français et en croate. Les adjectifs ont été relevés dans frWaC et $h r W a C$, deux corpus de très grande taille dont les données sont collectées sur Internet. Une analyse des bases a montré que, comme on pouvait s'y attendre, les préfixes analysés sont majoritairement utilisés dans des domaines spécialisés. L'analyse morphosémantique des significations réalisées par le préfixe $s u b$ - a montré qu'il s'agit dans les deux langues d'un préfixe polysémique dont les sens sont intimement liés et basés sur la métaphore et contiguïté conceptuelle. Ce fait soutient la thèse cognitiviste qu'au niveau sémantique les affixes dérivationnels sont proches des catégories traditionnellement appelées « lexicales ».

Mots-clés : français, croate, régularités morphosémantiques, préfixe, sub-

\section{Introduction}

La présente contribution traite d'une question particulière qui relève $\mathrm{du}$ domaine de la morphologie dérivationnelle, à savoir du rôle du préfixe latin sub-dans les lexiques français et croates, plus précisément dans la formation des adjectifs. Cette question, d'apparence assez simple, touche à plusieurs questions linguistiques additionnelles, dont notamment aux contacts linguistiques, à l'emprunt (morphologique), à la nature des affixes dérivationnels et à leur comportement sémantique.

Afin d'explorer la question annoncée au début, nous allons d'abord fournir un bref aperçu de la théorie des emprunts morphologiques, suivie d'un récapitulatif de l'influence du latin sur le français et le croate, pour ensuite proposer une analyse morphosémantique des adjectifs en question.

\section{L'emprunt linguistique}

L'emprunt est un phénomène linguistique quasi-universel (cf. Grant 2019: 1) qui influe sur la langue cible à de nombreux niveaux, notamment celui du lexique 
(e.g. Deroy 1956 ; Thomason 2001 ; Turk 2013). A la différence des emprunts lexicaux, les emprunts morphologiques ont été considérablement moins explorés, mais ont suscité un intérêt grandissant dans les dernières années (Gardani / Arkadiev / Amiridze 2015: 1).

Quand il s'agit des emprunts morphologiques, Grevisse / Goosse (1995: 57) soulignent que des langues de prestige, auxquelles on prend beaucoup de mots, donnent aussi d'autres éléments comme mots grammaticaux, suffixes et procédés de formation. Lorsqu'il s'agit de l'emprunt des préfixes, qui nous concerne plus particulièrement ici, Deroy (1956 : 70) a déjà remarqué qu'ils « s'empruntent sans trop de difficulté ». Il soutient l'idée que les préfixes étrangers sont dans la plupart des cas empruntés indirectement, par le biais de mots complexes dont ils font partie. A la différence de Deroy (1956), certains auteurs comme Seifart (2015) considèrent que les morphèmes peuvent être empruntés directement aussi, au cas où il existe un niveau de compréhension assez élevé entre les langues concernées.

\section{L'influence du latin}

Le préfixe $s u b$ - est un préfixe savant, l'équivalent du préfixe populaire français sous-, ${ }^{1}$ ainsi que du préfixe croate pod-. ${ }^{2}$

Le français a connu une forte latinisation pendant son histoire, empruntant en masse des mots latins, surtout depuis le 14 e siècle (Ullmann 1952: 127 ; Zwanenburg 1983 : 1). Toutefois, « [1'] influence du latin ne s'est jamais interrompue », surtout dans les sciences (Grevisse/Goosse 1995 : 57). Ce «latin scientifique et savant », comme l'appelle Helander (2010), est surtout utilisé, avec le grec, pour créer des mots nouveaux afin de nommer de nouveaux concepts qui accompagnent la croissance rapide des connaissances. A côté de l'emprunt de lexèmes et de bases, le latin a fourni aussi « un grand nombre de suffixes et de préfixes » (Pešek 2007 : 62), ainsi que des procédés dérivationnels (Zwanenburg 1983 : 1). Il faut souligner que ces éléments ou procédés empruntés peuvent « commencer à mener leur vie propre » (Zwanenburg 1983 : 5) une fois entrés dans la langue cible, devenant ainsi des « opérateurs dérivationnels autonomes » (Pešek 2007 : 62).

Ce dernier fait est très important pour cette contribution car il faut savoir qu'en français contemporain, le préfixe sub-forme grosso modo deux types de mots. D’un côté, il existe des formes dites « lexicalisées » qui ont été pour la plupart empruntées telles quelles au latin, et de l'autre il y a des formations françaises plus récentes (cf. TLFi), ${ }^{3}$ qui font partie de la néologie préfixale.

Le croate, quant à lui, a eu des contacts tout au long de son histoire avec le latin comme langue de la chrétienté d'Occident, ainsi que celle de l'administration

1 Voir https://www.cnrtl.fr/definition/sub-.

2 Voirhttp://hjp.znanje.hr/index.php?show=search_by_id\&id=d1plUBE\%3D\&keyword=sup.

3 Selon le TLFi (Trésor de la langue française informatisé), le préfixe sub-apparait dans « de nombreux » mots construits empruntés au latin, ainsi que dans « de nombreux mots de création française », dont les plus récents concernent surtout les domaines des sciences et technologies. Voir https://www.cnrtl.fr/definition/sub-. 
et de l'éducation (Samardžija 2002 : 61). Pendant une longue période s'étalant jusqu'au 19e siècle, dans les territoires de la Croatie continentale le latin était non seulement la langue du culte, mais aussi la langue officielle ${ }^{4}$ tandis que le croate s'utilisait dans les journaux, en littérature et dans la communication privée (Samardžija 2002 : 66). De nombreux mots nouveaux croates ont été formés à la base du latin (et du grec) surtout depuis la révolution industrielle (Samardžija $2002: 65)$.

\section{Cadre théorique}

Cet article repose sur le modèle des régularités morphosémantiques ${ }^{5}$ (Raffaelli 2013), qui attribue une égale importance aux procédés morphologiques et sémantiques dans la formation du lexique construit. Cela implique que chaque lexème motivé est considéré comme doublement motivé : morphologiquement et sémantiquement. Ce fait souligne également qu'entre le lexique et la grammaire il n'y a pas de frontières strictes, mais que les deux forment un continuum.

Si les mots complexes ont été pendant longtemps plutôt étudiés sous l'aspect grammatical, ou celui des procédés de la formation des mots, aujourd'hui on souligne de plus en plus leur aspect sémantique (e.g. Onysko / Michel 2010). En outre, certains auteurs (e.g. Raffaelli 2018: 153) soutiennent que l'un des défis majeurs de la formation des mots aujourd'hui en tant que discipline linguistique est justement l'étude systématique des processus sémantiques qui accompagnent la formation des mots complexes.

Les adjectifs analysés dans cette contribution sont formés selon deux procédés :

1) préfixation [sub-[Adj] $]_{\mathrm{Adj}}$ (subadulte, subaigu; subinteligentan 'subintelligent')

2) parasynthèse [sub-[N]-Suff $]_{\mathrm{Adj}}$ (subthalamique; subalpski 'subalpin')

\section{Méthodologie}

Deux corpus électroniques ont été utilisés afin de relever les adjectifs français et croates. Il s'agit de frWaC et $h r W a C$, deux corpus de très grande taille collectés sur Internet qui sont, quant à notre connaissance, les plus grands corpus disponibles pour les deux langues étudiées.

Les données ont été rassemblées par le biais de la requête [word=»sub. ${ }^{*}$ ] containing $\left[\operatorname{tag}=» A .^{*} »\right]$ qui cherche tous les adjectifs commençant par la suite graphique $s u b$. Ensuite, en recourant à l'outil «Frequency - Lemma », une liste de tous les adjectifs formés avec sub- a été obtenue selon leur fréquence dans le corpus, des plus aux moins fréquents. Seuls les adjectifs ayant une fréquence d'au moins 10 occurrences ont été considérés dans l'analyse. Ces deux listes ont

4 Le parlement croate a voté le 23 octobre 1847 le remplacement du latin par le croate comme langue officielle (Samardžija 2002 : 66).

5 En croate, model morfosemantičkih obrazaca. 
ensuite été manuellement vérifiées afin d'éliminer le bruit (fautes de frappe, mots appartenant à d'autres parties du discours, adjectifs formés par suffixation (e.g. substituable) et adjectifs " lexicalisés » mentionnés ci-dessus). ${ }^{6}$

Une fois que les deux listes ont été filtrées, nous avons obtenu 43 adjectifs français et 50 adjectifs croates.

Les adjectifs ont par la suite été analysés du point de vue sémantique. Plus précisément, ils ont été répartis selon les domaines sémantiques auxquels ils appartiennent : géographie, médecine, biologie, etc. Le préfixe $s u b$ - a ensuite été analysé lui aussi du point de vue sémantique afin de repérer les significations qu'il réalise avec les bases auxquelles il s'attache.

A la fin de l'analyse, nous avons proposé une comparaison des adjectifs français et croates, ainsi que des significations préfixales dans les deux langues.

\section{Résultats et analyse}

Quant à l'analyse des bases, les adjectifs français peuvent être répartis dans les catégories suivantes, comme indiqué sur le Diagramme 1 :

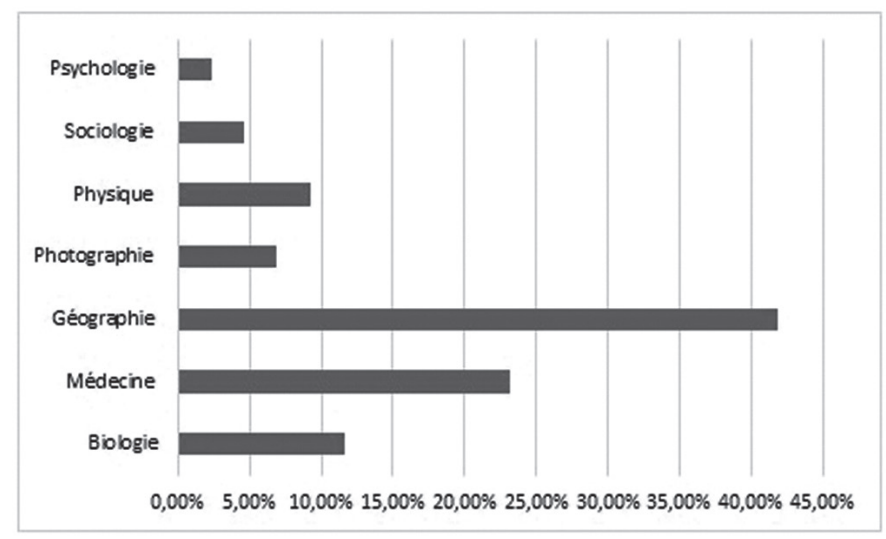

Diagramme 1 : Classification des bases françaises

Les bases croates montrent un comportement similaire, tout en englobant moins de catégories. La classification sémantique des bases en domaines de connaissances montre clairement que la plupart des adjectifs analysés sont des termes techniques, notamment scientifiques, qui ne font pas partie de la langue générale, mais de différentes langues de spécialité.

6 Il s'agit d'un assez grand nombre d'adjectifs tels que subséquent $<$ empr. au lat. subsequens>, sublime <empr. au lat. sublimis>, substitutif < empr. au b. lat. substitutivus>, etc. selon le TLFi. 


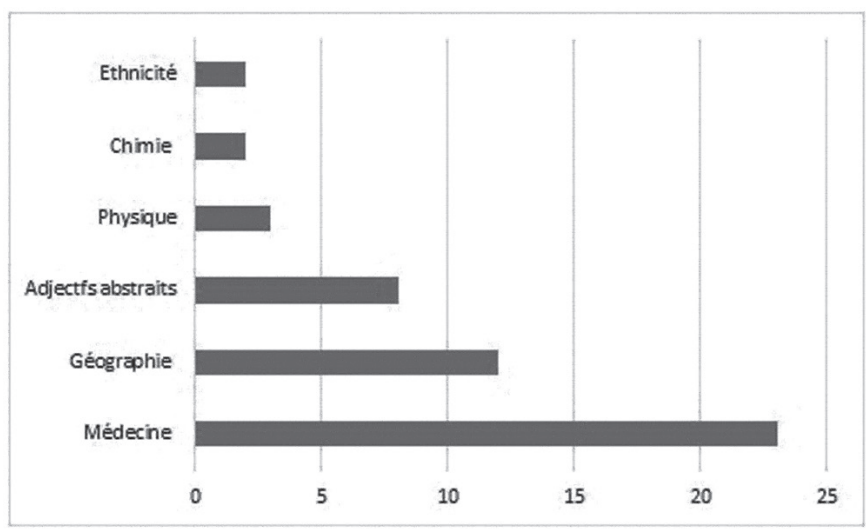

Diagramme 2 : Classification des bases croates $^{7}$

Le préfixe sub- quant à lui réalise les significations suivantes dans les adjectifs français :

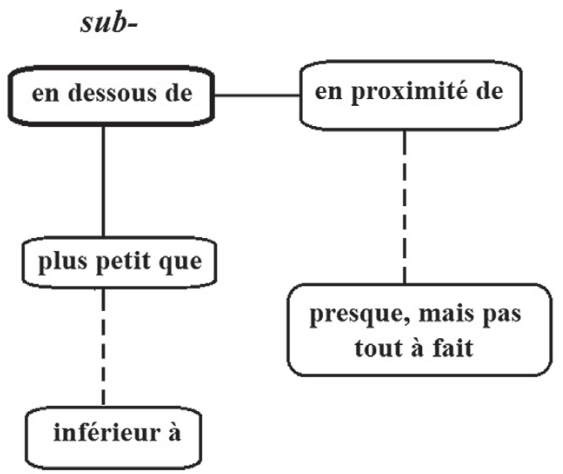

Image 1 : Réseau sémantique du préfixe sub- (adjectifs français)

Le réseau sémantique du préfixe $s u b$ - est composé de cinq significations étroitement liées par des liens de métaphore et de contiguïté conceptuelle. ${ }^{8}$ La signification 'en dessous de' est celle concrète, spatiale et prototypique ; on la trouve dans les adjectifs tels que subclavier 'qui est situé sous la clavicule', sublingual 'qui est situé sous la langue' et subaquatique 'qui a lieu sous l'eau'.

7 La catégorie « adjectifs abstraits » comporte trois adjectifs : subspecijaliziran 'sousspécialisé', subtotalan 'sous-total' et subinteligentan 'sous-intelligent'.

8 La contiguïté conceptuelle (ang. conceptual contiguity) est un processus de construction de sens liant deux éléments appartenant au même domaine. Le domaine (ang. domain) est pris ici dans le sens large du terme, comme indiquant toute structure de connaissances nécessaire pour la compréhension d'un mot quelconque (cf. Evans / Green 2006: 222). Si deux mots appartiennent au même domaine conceptuel, cela veut dire qu'il y a un lien extralinguistique entre eux. 
La signification 'en proximité de' est liée à la première à la base de contiguïté conceptuelle. Plus précisément, ce qui est 'en dessous de' quelque chose se trouve normalement 'en proximité de' telle chose. Les adjectifs basés sur ce principe sont, par exemple, subalpin, subarctique, subéquatorial, sublittoral 'qui est peu éloigné du littoral', etc.

La signification 'presque, mais pas tout à fait' est liée au sens prototypique par voie de la métaphore similarity is Closeness (e.g. Radden / Matthis 2002 : 232) selon laquelle on conceptualise des choses similaires comme étant physiquement proches. Les adjectifs basés sur cette métaphore sont, par exemple, subadulte '(d'un animal) qui n'est pas encore adulte', subaride 'dont les conditions climatiques sont proches de l'aridité', subdésertique 'dont les conditions sont proches de celles du désert' et sublétal 'presque mortel'.

Les deux significations 'plus petit que' et 'inférieur à' sont liées au sens prototypique 'en dessous de' par voie de métaphore. Il s'agit des métaphores LESS IS DOWN et LACK OF CONTROL IS DOWN (e.g. Lakoff 1987), respectivement, selon lesquelles des propriétés négatives comme moindre taille et infériorité sont conceptualisées comme étant dans une position inférieure dans l'espace. La première métaphore est à l'œuvre dans les adjectifs tels que subatomique, subcellulaire, sub-étatique et submétrique, et la seconde dans l'adjectif subhumain 'inférieur à l'homme'.

Avec les adjectifs croates, sub-réalise quatre significations : 'en dessous de' (e.g. subklavijski 'subclavier', subretinalan 'subretinien, qui est situé sous la rétine), 'plus petit que' (e.g. subatomski 'subatomique', subtotalan 'qui est presque total', submikronski 'submicronique'), 'en proximité de' (e.g. subpolaran 'subpolaire', subantarktički 'subantarctique') et 'presque, mais pas tout à fait' (subletalan 'sublétal', subfebrilan 'subfébrile'). Cela montre que le préfixe sub-construit des sens quasiment pareils avec les adjectifs français et croates, à l'exception du sens 'inférieur à' qui lui apparait juste dans l'adjectif français subhumain.

Ajoutons à la fin qu'il est possible que ces adjectifs soient des emprunts à l'anglais ou bien des formations sur le modèle anglais, vu l'influence globale de cette langue, notamment dans les sciences.

\section{Conclusion}

Comme le montre l'analyse des adjectifs français et croates formés avec le préfixe $s u b-$, ces lexèmes ne sont pas très nombreux en français et croate contemporains et s'emploient presque exclusivement dans des langues de spécialité, notamment dans les sciences telles que médecine, physique ou géographie. Cela n'est pas surprenant vu le fait que sub- est un préfixe savant. Toutefois, malgré leur nombre relativement restreint, l'analyse morphosémantique de ces adjectifs est importante car elle souligne et confirme deux faits. Premièrement, sub- est un préfixe polysémique créant un réseau sémantique systématisé basé sur extensions métaphoriques et contiguïté conceptuelle. Les relations qu'entretiennent les sens de ce préfixe imitent le modèle du prototype, avec le sens concret, spatial au centre, 
comme source de tous les autres sens. Secondement, cette organisation sémantique est très proche de celle des catégories traditionnellement dites « lexicales » (cf. Lehrer 2003). Ces deux faits soulignent l'importance de la dimension sémantique dans la formation des mots, et soutiennent l'idée de l'existence d'un continuum entre la grammaire et le lexique.

\section{Bibliographie}

Deroy, Louis (1956). L'emprunt linguistique, Paris : Les Belles lettres.

Evans, Vyvyan / Green, Melanie (2006). Cognitive Linguistics. An Introduction, Edimbourg : Edinburgh University Press.

Gardani, Francesco / Arkadiev, Peter / Amiridze, Nino (2015). Borrowed morphology: an overview, in : Borrowed Morphology [éds. Francesco Gardani / Peter Arkadiev / Nino Amiridze], Berlin / Boston / Munich : De Gruyter, pp. 1-24.

Grevisse, Maurice / André Goosse (1996). Nouvelle grammaire française, 3e édition, Bruxelles : De Boeck.

Helander, Hans (2010). Les fonctions du latin dans l'Europe moderne, https://journals. openedition.org/lettre-cdf/833 (10/03/2020).

Lakoff, George (1987). Women, Fire, and Dangerous Things: What Categories Reveal About the Mind, Chicago : University of Chicago Press.

Lehrer, Adrianne (2003). Polysemy in derivational affixes, in : Polysemy: Flexible Patterns of Meaning in Mind and Language [éds. Brigitte Nehrlich / Zazie Todd / Vimala Herman / David D. Clarke], Berlin: Mouton de Gruyter, pp. 217-232.

Onysko, Alexander / Michel, Sascha (2010). Unravelling the cognitive in word formation, in : Cognitive Perspectives on Word Formation [éds. Alexander Onysko / Sascha Michel], Berlin / New York : De Gruyter.

Pešek, Ondřej (2007). Enrichissement du lexique de l'ancien français : les emprunts au latin dans l'œuvre de Jean de Meun, in : Opera Universitatis Masarykianae Brunensis, 1, pp. 61-93.

Radden, Günter / Matthis, Elizabeth (2002). Why Similar To, but Different From?, in : Perspectives on Prepositions [éds. Hubert Cuyckens / Günter Radden], Tübingen : Max Niemeyer Verlag, pp. 231-256.

Raffaelli, Ida (2013). The model of morphosemantic patterns in the description of lexical architecture, in : Lingue e linguaggio, XII (1), pp. 47-71.

Raffaelli, Ida (2018). Kroz leksikologiju i semantiku, in : Od dvojbe do razdvojbe. Zbornik radova u čast profesorici Branki Tafri [éds. Petra Košutar / Mislav Kovačić], Zagreb : Ibis grafika.

Samardžija, Marko (2002). Nekoć i nedavno. Odabrane teme iz leksikologije i novije povijesti i hrvatskoga stadardnoga jezika. Rijeka : Izdavački centar Rijeka.

Seifart, Frank (2015). Direct and indirect affix borrowing, in : Language, 91, pp. 511-532.

Thomason, Sarah Grey (2001). Language contact: An introduction. Washington (DC) : Gerogetown University Press. 
Turk, Marija (2013). Jezično kalkiranje u teoriji i praksi. Prilog lingvistici jezičnih dodira. Zagreb: Hrvatska sveučilišna naklada / Filozofski fakultet u Rijeci. Ullmann, Stephen (1952). Précis de sémantique française. Berne : Francke. Zwanenburg, Wiecher (1983). Productivité morphologique et emprunt. Amsterdam / Philadelphia : John Benjamins.

\section{Morphosemantic analysis and comparison of adjectives formed with $s u b$ - in French and Croatian}

This paper analyses and compares adjectives formed with the prefix sub-in French and Croatian. The adjectives were retrieved from $\mathrm{frWaC}$ and $\mathrm{hrWaC}$, two large web corpora. An analysis of the bases has demonstrated that, unsurprisingly, the prefix sub-is mostly used in specialized domains. A morphosemantic analysis of the meanings realized by the prefix itself has shown that $s u b$ - is a polysemous prefix whose meanings are closely related and based on metaphorical extensions and conceptual contiguity. This fact only confirms the cognitive linguistics thesis that on the semantic level, derivational affixes are close to the categories traditionally dubbed as "lexical".

Key words: French, Croatian, morphosemantic patterns, prefix, sub- 